\title{
Prevalence, patterns, and factors associated with bleeding tendencies in dengue
}

\author{
Emmanuel Bhaskar, Gopalan Sowmya, Swathy Moorthy, Varun Sundar \\ Department of Medicine, Sri Ramachandra Medical College and Research Institute, Chennai, India
}

\begin{abstract}
Introduction: The pattern of bleeding tendencies in dengue and its corellation with platelet count and other factors requires clarification. Methodology: A retrospective study on bleeding tendencies in adults with dengue and platelet counts of less than 100,000 per $\mathrm{mm}^{3}$ was conducted. Factors associated with bleeding were analyzed. The study cohort were grouped as dengue with severe thrombocytopenia when platelet count was $<50,000 / \mathrm{mm}^{3}$ and as dengue with moderate thrombocytopenia if platelet count was $50,000-100,000 / \mathrm{mm}^{3}$

Results: A total of 638 patients formed the study cohort. A $24.1 \%$ prevalence of bleeding tendencies was observed. Prior anti-platelet drug intake, platelet count of $<70,000 / \mathrm{mm}^{3}$, international normalized ratio $>2.0$, and partial thromboplastin time $>60$ seconds were associated with bleeding. Esophagogastroduodenoscopy was found to identify structural gastroduodenal lesions when dengue was complicated by hematemesis or melena.

Conclusions: The results of this study suggest that bleeding complications in dengue can occur at platelet counts of up to $70,000 / \mathrm{mm}^{3}$, and that prior anti-platelet drug intake increases bleeding risk. Evaluation of hematemesis or melena in dengue with esophagogastroduodenoscopy is beneficial.
\end{abstract}

Key words: dengue; thrombocytopenia; bleeding; prevalence.

J Infect Dev Ctries 2015; 9(1):105-110. doi:10.3855/jidc.5031

(Received 24 May 2014 - Accepted 23 August 2014)

Copyright (C) 2015 Bhaskar et al. This is an open-access article distributed under the Creative Commons Attribution License, which permits unrestricted use, distribution, and reproduction in any medium, provided the original work is properly cited.

\section{Introduction}

Dengue disease has a global incidence of $50-100$ million cases annually and occurs among $30 \%-54.7 \%$ of the global population, spread over 128 countries whose inhabitants are at risk of acquiring the illness $[1,2]$. Though clear estimates of dengue are not available from India on account of poor reporting, it appears that dengue cases are on the rise. The Ministry of Health and Family Welfare reported a doubling of dengue cases in India in $2008(\mathrm{n}=12,419)$ compared to $2007(\mathrm{n}=5,534)$ [3]. Thrombocytopenia of varying severity is a consistent laboratory parameter in patients with dengue disease [4]. Bleeding tendencies in dengue are attributable to low platelet count, platelet dysfunction, activation of platelets, cytokines, vascular fragility, and coagulopathy $[5,6]$. The absolute platelet count that correlates with the occurrence of external or internal bleeding in dengue is not clear. Clinical studies on dengue among children and adults indicate a correlation between spontaneous bleeding and platelet count $<50,000 / \mathrm{mm}^{3}$ [7-10]. Our prior observation concurred with this cut-off for platelet count that relates to hemorrhagic manifestations in dengue [11]. These studies have often combined observations on bleeding tendencies in dengue illness across the entire platelet range below $100,000 / \mathrm{mm}^{3}$ and have occasionally categorized them into small subgroups (lower or higher range platelet count). Though reports have indicated the occurrence of bleeding tendencies at platelet counts between 50,000 and $100,000 / \mathrm{mm}^{3}$, there is no systematic observation on bleeding tendencies in this platelet range. A small number of studies observed no relation between bleeding and platelet count in dengue, but their conclusions are less convincing because they had very small sample sizes; furthermore, the spectrum of bleeding tendencies observed were not representative of those seen in clinical practice and lacked many clinical variables on analysis $[12,13]$. Furthermore, the information on absolute platelet count at the time of internal bleeding, duration of bleeding, severity of coexistent defect in coagulation pathway, medication factors, and patient factors are far from complete in most published reports. In the absence of a consensus on grading severity of thrombocytopenia in dengue, we defined a platelet count of 50,000 to $100,000 / \mathrm{mm}^{3}$ 
as moderate thrombocytopenia and a platelet count of less than $50,000 / \mathrm{mm}^{3}$ as severe thrombocytopenia and designed this study to observe the prevalence of bleeding tendencies in this entire range of platelet count, to identify if an absolute platelet level correlates with the occurrence of external or internal bleeding, and to assess factors co-existing with thrombocytopenia that could increase bleeding risk.

\section{Methodology}

Study design and participants

This was a retrospective study of patients hospitalized with a diagnosis of dengue disease between June 2010 and May 2013 at a tertiary care teaching hospital of Sri Ramachandra University located in Chennai, a metropolitan city in southern India. The study was approved by the institutional ethics committee. Medical records were perused by one of the study investigators to identify records of participants satisfying the 2009 World Health Organization (WHO)'s evaluation classification for dengue along with positive serology for acute dengue infection using an IgM and IgG capture enzyme-linked immunosorbent assay (ELISA) kit (Panbio, Brisbane, Australia) [14]. The subjects for the present study were required to be $\geq 18$ years of age, to satisfy WHO criteria for dengue, and to have no co-infections.

\section{Data collection}

Data were collected by manual perusal of records and were documented in a structured data collection sheet. The following information was recorded: age; gender; duration of hospitalization; place of hospitalization (ward or intensive care); symptoms of bleeding tendencies prior and during hospital admission; signs of cutaneous and mucosal bleeding tendencies; hemodynamic assessment; medications in nurse reconciliation form, especially anti-platelets drugs such as aspirin or clopidogrel and non-steroidal anti-inflammatory drug (NSAID); prior medical comorbidities; investigations on the day of hospitalization and during hospital stay (complete blood count, prothrombin time, partial thromboplastin time, serum creatinine, liver function tests, chest Xray, sonography of abdomen), and relevant investigations on the day of bleeding which included platelet count, international normalized ratio (INR) and partial thromboplastin time (PTT); investigations related to evaluation of possible dual infection (blood culture, urine culture, tests for malaria, scrub typhus, and leptospirosis); course of bleeding tendencies that were present on hospital admission and those which occurred after admission; investigations done to evaluate source of internal bleeding; blood products administered; medications received during hospitalization; and final outcome. Participants were classified as dengue \pm warning signs and severe dengue based on WHO guidelines [14]. Patients were further divided into two groups, based on severity of thrombocytopenia, as dengue with severe thrombocytopenia (DST) with a platelet count of $<$ $50,000 / \mathrm{mm}^{3}$, and dengue with moderate thrombocytopenia (DMT) with a platelet count of 50,000 to $100,000 / \mathrm{mm}^{3}$. Platelet count on admission among participants who presented with bleeding at initial hospitalization and platelet count on the day of bleeding (before administration of blood products) among participants who developed bleeding after hospitalization was defined as peri-bleeding platelet count. Prior anti-platelet drug intake was defined as documented chronic intake of aspirin or clopidogrel on the nurse reconciliation form.

\section{Statistical analysis}

Continuous variables were expressed as mean \pm standard deviation, and discrete variables were expressed as number (\%). Association between bleeding risk and patient factors (age $>60$ years, presence of prior chronic medical illness, chronic antiplatelet drug intake, recent NSAID intake, peribleeding platelet count, and coagulation parameters of varying ranges) were analyzed by univariate analysis; those which had a $p$ value $<0.2$ were analyzed by multiple logistic regression, which determined the possible cut-off for platelet count, INR, and PTT associated with bleeding using sensitivity analysis, and were adjusted for influence of individual variables. Analysis was done for the entire study cohort (both DST and DMT groups), and for the DMT group separately, following the mentioned plan. A p value $<$ 0.05 was considered to be statistically significant. Analysis was done with SPSS version 19.1.

\section{Results}

A total of 638 patients satisfied the study criteria and were included in the analysis. Fifty-two participants who had co-infections were excluded. Baseline and laboratory characteristics of DST $(\mathrm{n}=$ 246) and DMT $(n=392)$ patients are shown in Table 1. Thirty-two patients had platelet counts of less than $20,000 / \mathrm{mm}^{3}$. Four hundred and ninety-two patients were 35 years of age or younger. Forty were over 60 years of age. 
Table 1. Baseline and laboratory characteristics of study participants

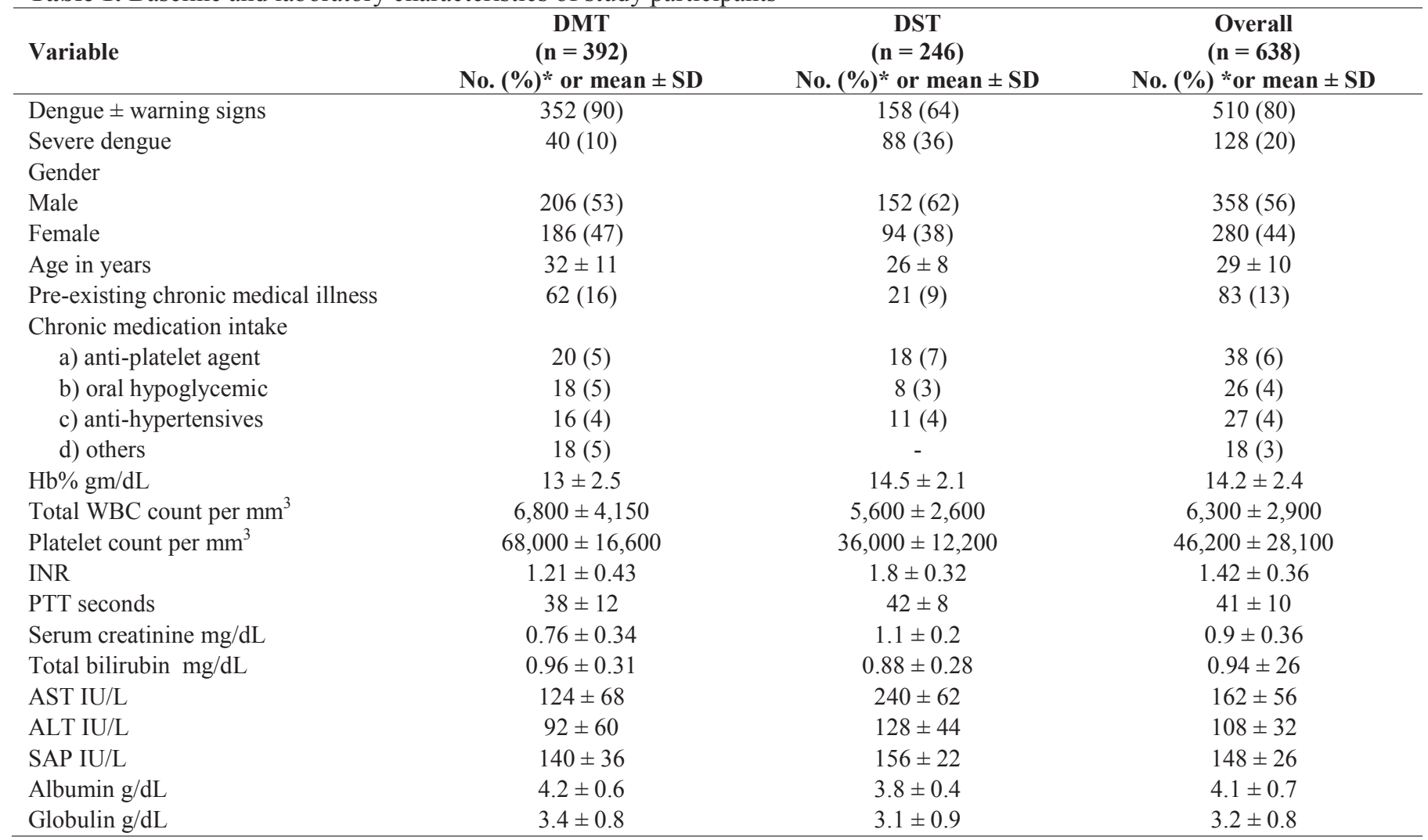

*Percentage rounded to nearest whole number

DMT: dengue with moderate thrombocytopenia; DST: dengue with severe thrombocytopenia; WBC: white blood cell; INR: international normalized ratio; PTT: partial thromboplastin time; AST: aspartate transaminase; ALT: alanine transaminase; SAP: serum alkaline phosphatase

Table 2. Pattern of bleeding tendencies with corresponding mean platelet count

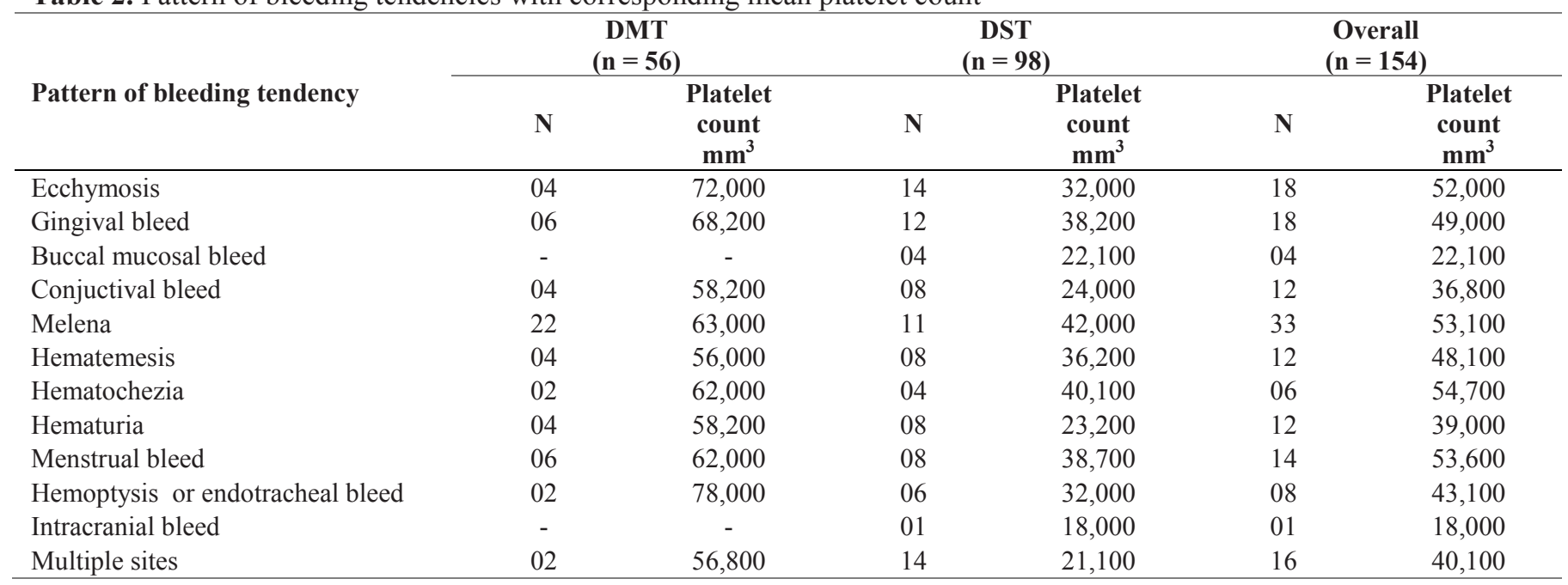


The prior chronic medical illnesses (CMIs) present in 83 participants included diabetes mellitus $(\mathrm{n}=30)$, hypertension $(\mathrm{n}=30)$, chronic kidney disease $(\mathrm{n}=16)$, coronary artery disease $(\mathrm{n}=16)$, bronchial asthma $(\mathrm{n}=$ $12)$, chronic obstructive pulmonary disease $(n=8)$, and others $(n=6)$. Fifty-five of the patients were hospitalized in wards, 66 in observation units, and 22 in the intensive care unit. Four hundred seventy-two patients received outpatient medical consultation prior to hospitalization and had taken NSAIDs $(n=58)$, antibiotics $(\mathrm{n}=242)$, or acid suppressants $(\mathrm{n}=174)$. Chest X-rays were normal in 502 patients, and 136 had features of mild pleural effusion. Sonography was done for 502 patients and was normal in 252, while it showed either altered gall bladder echoes or serositis $(\mathrm{n}=164)$ or both $(\mathrm{n}=86)$ in the other 250 patients.

One hundred fifty-four patients had bleeding tendencies, of which 121 had features of bleeding on admission, and 33 developed new onset bleeding after hospitalization. One hundred thirty-eight had bleeding at a single organ location. Table 2 shows patterns of bleeding tendencies and corresponding platelet counts observed in the study cohort. Platelet counts at the time of bleeding tendency were $41,300 \pm 18,100 / \mathrm{mm}^{3}$, INR was $1.3 \pm 0.84$, and PTT was $48 \pm 18$ seconds. Timing of bleeding during the course of illness was as follows: day $4(\mathrm{n}=12)$, day $5(\mathrm{n}=21)$, day $6(\mathrm{n}=22)$, day $7(n=46)$, day $8(n=41)$, day $9(n=6)$, and day $10(\mathrm{n}=6)$. Twelve of 58 participants with recent NSAID intake had bleeding (six with gingival bleeding, two with hematemesis, two with ecchymosis, and two with melena). Twenty-four of 38 participants on chronic anti-platelet drug intake had bleeding (eight with melena, four with hematemesis, two with hematuria, one with hematochezia, four with ecchymosis, and five with gingival bleeding). Twenty- four of 33 patients with melena underwent esophagogastroduodenoscopy (EGD), which showed diffuse erythematous gastric mucosa $(n=12)$, gastric ulcer with evidence of recent bleed $(n=7)$, duodenal ulcer with evidence of recent bleed $(n=3)$, gastric carcinoma $(n=1)$, and normal findings $(n=1)$. All 12 patients who had hematemesis underwent EGD, which showed features of erosive gastropathy $(\mathrm{n}=10)$ and gastric ulcers $(n=2)$. Since active ulcer bleeding was not found during endoscopy, no intervention (such as clipping) was done. Gingival, gastrointestinal, genitourinary bleeding, and hemoptysis lasted for a mean of three days (range, one to five days). Four patients with hematemesis (mean platelet count of $22,000 / \mathrm{mm}^{3}$ ) and one each with hematuria (platelet count of $24,200 / \mathrm{mm}^{3}$ ) and menorrhagia (platelet count of $19,800 / \mathrm{mm}^{3}$ ) received whole blood transfusions since bleeding persisted beyond 24 hours and the volume of blood loss warranted replacement. Bleeding tendencies did not result in hemodynamic compromise. Chest X-rays were normal in patients who suffered hemoptysis. No progression of ecchymosis $(n=18)$ was observed. Evaluation for hematuria and increased menstrual bleed was not done. Ninety-six patients received platelet concentrates (median, two; range, one to eight), and 12 received one unit of single donor platelets. Seventytwo of 108 participants who were transfused with platelet products had an increase in platelet count $\left(23,000 \pm 8,000 / \mathrm{mm}^{3}\right), 34$ had no increase, and two had marginal $\left(8,000-10,000 / \mathrm{mm}^{3}\right)$ falls in platelet count. Mean hospital stay was 6.5 days (range, 2 to 16 days). Patients with bleeding at admission or after hospitalization were observed for a minimum of three days. Eighteen patients, all in the DST group, expired. None of the expired patients had persistent bleeding at

Table 3. Risk factors for bleeding among study participants

\begin{tabular}{|c|c|c|c|c|}
\hline \multirow{2}{*}{ Variable } & \multirow{2}{*}{ Odds ratio } & \multicolumn{2}{|c|}{ 95\% Confidence interval } & \multirow{2}{*}{$P$ value } \\
\hline & & Lower & Upper & \\
\hline \multicolumn{5}{|l|}{ Entire cohort } \\
\hline Prior anti-platelet intake & 2.62 & 1.31 & 3.95 & 0.003 \\
\hline Platelet $<70,000 / \mathrm{mm}^{3}$ & 1.62 & 1.08 & 2.86 & 0.002 \\
\hline $\mathrm{INR}>2.0$ & 1.86 & 1.11 & 2.36 & 0.021 \\
\hline PTT $>60$ seconds & 1.61 & 0.92 & 2.17 & 0.031 \\
\hline Prior anti-platelet drug intake & 2.08 & 1.21 & 3.16 & 0.005 \\
\hline NSAID intake & 0.86 & 0.57 & 1.52 & 0.17 \\
\hline Platelet $<70,000 / \mathrm{mm}^{3}$ & 1.92 & 1.12 & 2.86 & 0.007 \\
\hline $\mathrm{INR}>1.5$ & 0.78 & 0.47 & 1.32 & 0.17 \\
\hline PTT $>60$ seconds & 0.87 & 0.59 & 0.91 & 0.23 \\
\hline
\end{tabular}

NSAID: non-steroidal anti-inflammatory drug 
the time of death.

Univariate analysis of the whole cohort identified prior anti-platelet drug use $(\mathrm{p}=0.007)$, peri-bleeding platelet count of less than $70,000 / \mathrm{mm}^{3}(\mathrm{p}=0.02)$, INR $>2.0(\mathrm{p}=0.03)$, and PTT $>60$ seconds $(\mathrm{p}=0.01)$ to be significantly associated with bleeding tendency, while age $>60$ years $(p=0.28)$, gender $(p=0.43)$, presence of prior chronic medical illness $(p=0.24)$, and recent NSAID use $(p=0.21)$ were not associated with bleeding. However, multiple logistic regression, which adjusted for possible similar linear effects of independent variables on bleeding, identified prior anti-platelet drug intake $(p=0.003)$, peri-bleeding platelet count of less than $70,000 / \mathrm{mm}^{3}(\mathrm{p}=0.002)$, INR $>2.0(\mathrm{p}=0.021)$, and PTT $>60$ seconds $(\mathrm{p}=$ 0.031 ) as significant factors associated with bleeding tendency (Table 3).

Univariate analysis of the DMT group identified prior anti-platelet drug use $(p=0.004)$ and peribleeding platelet count of less than $70,000 / \mathrm{mm}^{3}$ ( $\mathrm{p}=$ $0.017)$ to be significantly associated with bleeding tendency, while age $>60$ years $(\mathrm{p}=0.36)$, gender $(\mathrm{p}=$ $0.57)$, presence of prior chronic medical illness $(p=$ $0.26)$, recent NSAID use $(\mathrm{p}=0.19)$, INR $>1.5(\mathrm{p}=$ $0.18)$, and PTT $>60$ seconds $(\mathrm{p}=0.14)$ were not associated with bleeding. Multiple logistic regression identified prior anti-platelet drug intake $(p=0.005)$ and peri-bleeding platelet count of less than $70,000 / \mathrm{mm}^{3}$ ( $\left.\mathrm{p}=0.007\right)$ as the significant factors associated with bleeding tendency (Table 3 ).

\section{Discussion}

The present study observed a $24.1 \%$ overall prevalence of bleeding tendencies in patients with dengue disease $(39.8 \%$ in DST and $14.2 \%$ in DMT). Seventy-four percent of bleeding tendencies occurred between day 6 and day 8 of dengue illness. Gastrointestinal bleeding (33.1\%) was as common as muco-cutaneous bleeding (33.7\%), which could be due to higher prevalence of asymptomatic gastroduodenal lesions in our study cohort. Prior anti-platelet drug intake, platelet count of $<70,000 / \mathrm{mm}^{3}$, INR $>2.0$, and PTT $>60$ seconds were significantly associated with the occurrence of bleeding. Risk of bleeding attributable to prior anti-platelet drug intake and platelet count of $<70,000 / \mathrm{mm}^{3}$ persisted in the DMT group. These observations indicate that a platelet count of $<70,000 / \mathrm{mm}^{3}$ is associated with bleeding independent of other factors. This finding differs from previous studies that observed that bleeding tendencies in dengue often correlate with a platelet count of $<$ $50,000 / \mathrm{mm}^{3}$ [7-10]. However, except for one study that had a similar research aim to ours of correlating thrombocytopenia with bleeding, other studies made the observation as part of a larger objective and hence did not document platelet count and coagulation parameters at the time of bleeding [7-10]. The spectrum of bleeding tendencies observed in our study was wide, unlike in previous studies where genitourinary or upper gastrointestinal bleed was not observed in the entire study cohort [12,15]. Our practice of simultaneous testing of platelet count and coagulation profile (INR and PTT) at the time of bleeding enabled us to appreciate the causal association of thrombocytopenia and severity of coagulopathy with bleeding tendencies.

In this study, asymptomatic structural gastroduodenal lesions $(n=13)$ were uncovered by dengue illness as a result of evaluation for melena $(\mathrm{n}=$ $11)$ or hematemesis $(n=2)$. The identification of gastroduodenal lesions in the DMT group resulted from clinicians' judgments that moderate thrombocytopenia may not result in melena or hematemesis in the absence of prior gastroduodenal lesions. It may be observed that this practice of evaluation was not followed for 9 of 33 patients with melena, indicating variation in clinical practice. Furthermore, patients who also had genitourinary bleed did not undergo further evaluation for possible pre-existing organ pathology. In patients with normal platelet counts and coagulation profiles, upper gastrointestinal endoscopy is considered to be a standard of care in the evaluation of melena or hematemesis. Current guidelines on dengue do not discuss the need for such evaluation, and the present study can form the basis for scientific enquiry into this topic.

Prior anti-platelet drug intake and not recent NSAID intake resulted in bleeding tendencies; this correlation reached statistical significance in our study. Chronic intake of anti-platelet drugs or NSAIDs are associated with high risk of upper gastrointestinal bleeding [16]. The bleeding risk with short-term exposure to these medications, however, appears to be low [16]. Though the observations in this study are in concurrence with the literature on bleeding risk in patients on chronic anti-platelet medication and patients using NSAIDs short term, the inference does not imply that short-term exposure to NSAIDs is safe in dengue illness complicated by thrombocytopenia. Our retrospective study design calls for a prospective observation of the effects of short-term NSAID exposure on dengue. 


\section{Conclusions}

The results of our study revealed that internal bleeding tendencies can occur in dengue illness associated independently with platelet counts of less than $70,000 / \mathrm{mm}^{3}$ and that prior anti-platelet drug intake increases bleeding risk. When upper gastrointestinal bleeding is associated with dengue, evaluation with EGD is beneficial in detecting prior asymptomatic gastroduodenal lesions. A prospective study to identify clinical predictors of structural lesions in EGD among dengue patients with gastrointestinal bleeding will give more clarity on the topic. Further data is required to determine the benefits of evaluating hematuria, menorrhagia, and hemoptysis in this setting.

\section{Acknowledgements}

We thank Mr. Singarayar and Ms. Janes of the Medical Records Department, Sri Ramachandra University, for helping us acquire the case sheets for perusal.

\section{References}

1. Wilder-Smith A, Macary P (2014) Dengue: challenges for policy makers and vaccine developers. Curr Infect Dis Rep 16: 404.

2. Brady OJ, Gething PW, Bhatt S, Messina JP, Brownstein JS, Hoen AG, Moyes CL, Farlow AW, Scott TW, Hay SI (2012) Refining the Global Spatial Limits of Dengue Virus Transmission by Evidence-Based Consensus. PLoS Negl Trop Dis 6: e1760.

3. Baghchi S (2009) Dengue bites India. CMAJ 180: E7.

4. Gubler DJ (1998) Dengue and dengue hemorrhagic fever. Clin Microbiol Rev 11: 480-496.

5. Schexneider KI, Reedy EA (2005) Thrombocytopenia in dengue fever. Curr Hematol Rep 4: 145-148.

6. Simmons CP, Farrar JJ, Nguyen vV, Wills B (2012) Dengue. N Engl J Med 366: 1423-1432.

7. Shivbalan S, Anandnathan K, Balasubramanian S, Datta M, Amalraj E (2004) Predictors of spontaneous bleeding in Dengue. Indian J Pediatr 71: 33-36.

8. Balmaseda A, Hammond SN, Pérez MA, Cuadra R, Solano S, Rocha J, Idiaquez W, Harris E (2005) Short report: assessment of the World Health Organization scheme for classification of dengue severity in Nicaragua. Am J Trop Med Hyg 73: 1059-1062.

9. Malavige GN, Velathanthiri VG, Wijewickrama ES, Fernando S, Jayaratne SD, Aaskov J (2006) Patterns of disease among adults hospitalized with dengue infections. QJM 99: 299-305.

10. Makroo RN, Raina V, Kumar P, Kanth RK (2007) Role of platelet transfusion in the management of dengue patients in a tertiary care hospital. Asian J Transfus Sci 1: 4-7.

11. Bhaskar ME, Moorthy S, Kumar NS, Arthur P (2010) Dengue haemorrhagic fever among adults - an observational study in Chennai, south India. Indian J Med Res 132: 738-740.

12. Krishnamurti C, Kalayanarooj S, Cutting MA, Peat RA, Rothwell SW, Reid TJ, Green S, Nisalak A, Endy TP, Vaughn DW, Nimmannitya S, Innis BL (2001) Mechanisms of hemorrhage in dengue without circulatory collapse. Am J Trop Med Hyg 65: 840-847.

13. Mourão MP1, Lacerda MV, Macedo VO, Santos JB (2007) Thrombocytopenia in patients with dengue virus infection in the Brazilian Amazon. Platelets 18: 605-612.

14. World Health Organization (2009) Dengue: guidelines for diagnosis, treatment, prevention and control. Geneva: WHO. Available:

http://www.who.int/tdr/publications/documents/denguediagnosis.pdf. Accessed May 23, 2014

15. Hammond SN, Balmaseda A, Pérez L, Tellez Y, Saborío SI, Mercado JC, Videa E, Rodriguez Y, Pérez MA, Cuadra R, Solano S, Rocha J, Idiaquez W, Gonzalez A, Harris E (2005) Differences in dengue severity in infants, children, and adults in a 3-year hospital-based study in Nicaragua. Am J Trop Med Hyg 73: 1063-1070.

16. Sostres C, Gargallo CJ, Lanas A (2013) Nonsteroidal antiinflammatory drugs and upper and lower gastrointestinal mucosal damage. Arthritis Res Ther 15 Suppl 3: S3.

\section{Corresponding author}

Emmanuel Bhaskar, MD

Professor, Department of Medicine

Sri Ramachandra Medical College and Research Institute

Porur, Chennai-600116

Tel: +91-44-26211056

Mobile: +919840717971

Email: drmeb1974@yahoo.co.in

Conflict of interests: No conflict of interests is declared. 